\title{
Philosophiques
}

\section{Moment cinétique et syllogistique dynamique chez Hegel}

\section{Yvon Gauthier}

Volume 32, numéro 2, automne 2005

URI : https://id.erudit.org/iderudit/011872ar

DOI : https://doi.org/10.7202/011872ar

Aller au sommaire du numéro

Éditeur(s)

Société de philosophie du Québec

ISSN

0316-2923 (imprimé)

1492-1391 (numérique)

Découvrir la revue

Citer cet article

Gauthier, Y. (2005). Moment cinétique et syllogistique dynamique chez Hegel. Philosophiques, 32(2), 357-368. https://doi.org/10.7202/011872ar

\section{Résumé de l'article}

Le terme de moment est omniprésent dans l'oeuvre de Hegel, et les commentateurs n'ont pas suffisamment insisté sur le sens dynamique du "Moment » hégélien qui n’a rien de temporel, mais dénote plutôt le momentum ou moment cinétique de la mécanique newtonienne. Hegel a donné vie à ce concept de moment et en a fait le moteur de sa dialectique qu'on interprète ici comme une syllogistique dynamique de la sursomption des moments du procès de la conscience et du devenir de l'esprit. Une logique dynamique pourrait récupérer avantageusement cette dialectique des concepts. Mais la lecture critique de Hegel veut montrer comment un concept physique est transformé en notion métaphysique et comment une science de la logique "Wissenschaft der Logik » est dévoyée dans une ontologie où c'est une philosophie de la nature qui devient mécanique en assujettissant la physique à un idéalisme objectif supraphysique. Un épilogue sur le vocabulaire hégélien termine l'article. 


\title{
Moment cinétique et syllogistique dynamique chez Hegel
}

\author{
YVON GAUTHIER \\ Université de Montréal \\ yvon.gauthier@umontreal.ca
}

\begin{abstract}
RÉSUMÉ. - Le terme de moment est omniprésent dans l'œuvre de Hegel, et les commentateurs n'ont pas suffisamment insisté sur le sens dynamique du «Moment» hégélien qui n'a rien de temporel, mais dénote plutôt le momentum ou moment cinétique de la mécanique newtonienne. Hegel a donné vie à ce concept de moment et en a fait le moteur de sa dialectique qu'on interprète ici comme une syllogistique dynamique de la sursomption des moments du procès de la conscience et du devenir de l'esprit. Une logique dynamique pourrait récupérer avantageusement cette dialectique des concepts. Mais la lecture critique de Hegel veut montrer comment un concept physique est transformé en notion métaphysique et comment une science de la logique «Wissenschaft der Logik» est dévoyée dans une ontologie où c'est une philosophie de la nature qui devient mécanique en assujettissant la physique à un idéalisme objectif supraphysique. Un épilogue sur le vocabulaire hégélien termine l'article.
\end{abstract}

\begin{abstract}
The readers of Hegel fail invariably to notice that the ubiquitous term "Moment" in Hegel's works does not refer to a temporal process, but to a dynamics of motion which has its origin in the Newtonian definition of momentum for material bodies. Hegel's notion of Moment is rather the momentum of a Spirit which is the driving force of a dialectical process described here as dynamical syllogistics. The dynamical logic of concepts could salvage what is still alive in Hegelian dialectics. Finally, critical reading of Hegel's philosophy shows how a physical concept is endowed with metaphysical meaning and how a science of logic "Wissenschaft der Logik" is transformed into ontology. Here it is a philosophy of nature which is substituted to the science of mechanics in the transformation of physics into a supraphysical objective idealism. The paper ends with an epilogue on Hegel's vocabulary.
\end{abstract}

\section{Introduction}

\section{Le concept de moment}

Pour le logicien et le philosophe des sciences contemporain, la logique et la philosophie de la nature de Hegel offrent peu d'intérêt sur le plan formel. Si la philosophie de la nature de Hegel n'est plus qu'une curiosité historique aux yeux du philosophe des sciences, la logique de Hegel peut être réhabilitée non pas comme logique dialectique au sens que la tradition marxiste a voulu donner à ce mot, mais comme ce que j'appellerai la syllogistique dynamique ou logique dynamique au sens où on l'entend maintenant en informatique théorique et en théorie de l'intelligence artificielle. "Intelligence artificielle " peut sembler détonner dans un contexte hégélien. Mais Hegel lui-même n’a 
pas manqué de noter que la philosophie a besoin d'une langue artificielle "Kunstsprache» pour rendre les déterminations réflexives «reflektierte Bestimmungen ", comme il dit dans la Science de la logique ${ }^{1}$. Et Hegel d'ajouter que le latin, à défaut de la langue maternelle, fournit souvent la terminologie nécessaire à la conceptualisation philosophique.

J'en veux pour exemple le terme de "Moment», un des termes les plus courants dans le texte de Hegel. Ce n'est pas le sens temporel du terme qui prédomine chez Hegel, mais le sens physique ou "dynamique» du latin "momentum» qui signifie, comme chacun sait, quantité de mouvement en physique classique et en mécanique newtonienne. Il est significatif à cet égard que Hegel emploie presque toujours le neutre "das Moment» pour signifier l'aspect dynamique du moment ou momentum, et il n'y pratiquement pas d'occurrences du terme dans le texte hégélien où ce sens serait absent. Hegel connaissait évidemment le sens physique du «momentum» ou moment cinétique chez Newton aussi bien que le Kant de la Critique de la raison pure qui le définit dans la deuxième analogie de l'expérience comme l'opération continue et uniforme de la causalité: eine kontinuierliche Handlung der Kausalität, welche, sofern sie gleichformig ist, ein Moment heisst ( "l'action continue de la causalité s'appelle moment dans la mesure où elle est uniforme $\left.{ }^{2} »\right)$.

Kant dit alors que le changement n'est pas constitué de moments, mais qu'il est causé par l'effet de ces moments. Ce sens n'est pas étranger à Hegel qui avait bien lu Newton avant de le critiquer pour son mécanisme, mais il transforme le sens physique initial pour lui donner une autre dimension sémantique, celle de moments dans l'auto-mouvement de l'esprit. C'est ici précisément que l'on pourrait dire que Hegel transmue la physique newtonienne en une métaphysique cinétique de l'esprit dans son idéalisme objectif.

Peu de commentateurs ont noté que l'allemand «Moment» a le sens de «momentum» que Hegel a tiré de sa lecture de Newton. Je rappelle que Newton définit les moments de quantités (physiques): Momenta quantitatum sunt ipsarum principia generantia vel alterantia fluxo continuo ( Les moments des quantités sont les principes de génération ou d'altération de ces mêmes quantités dans un flux continu $\left.{ }^{3} »\right)$.

La seconde loi de Newton définit le moment newtonien comme le produit de la masse et de la vitesse:

$$
\mathrm{p}=\mathrm{mv}
$$

et la célèbre formule de Newton pour la force donne:

$$
\mathrm{F}=\mathrm{ma}
$$

où $a$ est l'accélération.

1. Wissenschaft der Logik [7] p. 94-95

2. Kant, Critique de la raison pure. Voir 14, B. 254.

3. Hegel, 15, vol. VI, 192. 
Or Hegel, qui multiplie les emplois de «Moment» comme dans les expressions "Momente des Prozesses", "Momente des Werdens" ou "Momente des Begriffs ", y réfère toujours en termes d'un principe générateur ou modificateur d'un mouvement ou d'un flux continu dans la terminologie de Newton - notons ici que c'est chez Newton que "momentum» acquiert son sens plein de principe du mouvement, plutôt que de quantité de mouvement comme l'avaient conçu ses prédécesseurs, en particulier Descartes à qui l'on doit la première formulation du concept. Cet usage est encore plus net dans l'exemple du levier "Hebel» que Hegel utilise comme véritable paradigme ou modèle dynamique de la "sursomption", ma traduction pour "Aufhebung». Dans la Science de la logique, il nous dit:

Etwas ist nur insofern aufgehoben als es in die Einheit mit seinen Entgegengesetzten getreten ist; in diesen nähern Bestimmung als ein Reflektiertes kann es passend Moment genannt werden ${ }^{4}$ ( Q Quelque chose n'est sursumée que dans la mesure où elle est unie à son contraire; en tant que réfléchie dans cette détermination plus précise, elle peut être appelée moment.»)

La sursomption est opérée comme momentum dans l'unité des contraires, c'est-à-dire comme ce mouvement même de la réconciliation des forces opposées. Hegel donne tout de suite après ce passage l'exemple du levier où moment cinétique angulaire, ou encore moment d'un couple de forces sont les moteurs d'une dialectique à la fois concrète et abstraite. Le moment angulaire a la formule:

$$
\mathrm{L}=\mathrm{rp}
$$

$r$ pour le rayon et $p$ pour le moment cinétique.

Mais c'est dans la mécanique de la philosophie de la nature de Iéna qu'on trouve les passages les plus explicites sur la sursomption des moments dans le modèle du levier: Im Hebel sind alle Momente der Bewegung als einer aufgehobenen und sie als solche realisiert ( "dans le levier sont réalisés tous les moments du mouvement comme sursumés dans la sursomption même du mouvement»).

On connaît l'effort spéculatif de Hegel pour élever le processus mécanique du levier à la dignité de l'auto-mouvement du concept «die Selbstbewegung des Begriffs", mais il nous suffit de dire que le levier a été pour Hegel le modèle canonique de la sursomption dialectique du mouvement dans l'unité de ses moments. C'est à une véritable dynamique de l'esprit plutôt qu'à une cinétique des corps en mouvement que Hegel a voulu consacrer l'essentiel de sa logique. C'est ce que je veux essayer de montrer maintenant en mettant l'accent sur ce que j'appelle la syllogistique dynamique.

Doit-on réévaluer la logique dialectique à la lumière des développements récents en logique philosophique après les résultats négatifs de [3] et [4] du

4. Science de la logique, 7, 94.

5. Iéna, 8, 252. 
point de vue formel de la logique mathématique? Certains auteurs, comme da Costa, voient dans la logique dialectique une logique paraconsistante capable d'accueillir en son sein la contradiction tout en demeurant consistante, mais je pense qu'il est plus approprié de traiter la logique de Hegel comme une logique dynamique du syllogisme. Je définis plus loin un système particulier de logique non monotone compatible avec une théorie du raisonnement dynamique apte à rendre compte de la conception hégélienne de l'automotion du concept $<$ Selbsbewegung des Begriffs $>$.

\section{La logique dynamique}

En un mot, la logique dynamique est une logique des propositions (ou énoncés) et des actions; cette logique est d'abord apparue en programmation logique et informatique théorique où une machine représente des objets linguistiques et les manipule. Assurément, on doit élargir considérablement ce cadre pour y faire entrer la logique hégélienne en tant que théorie de l'action ou travail du concept <die Arbeit des Begriffs $>$, comme le dit Hegel à la fin de la préface de la Phénoménologie de l'esprit. Si l'on admet cet élargissement, j'utiliserai le terme de «logique dynamique» pour désigner une logique qui tente de formaliser la dynamique de l'action ou de l'activité conceptuelle.

La logique dynamique est essentiellement une logique de la représentation de la croissance du savoir. À ce chapitre, il y a plusieurs entrées dans le paysage logique contemporain, mais la cible principale est de formuler un système logique capable de formaliser l'idée de développement. On est encore loin de la notion d'<Aufhebung> que j'ai traduite par «sursomption» comme antonyme de la subsomption kantienne. La logique dynamique est parente de la logique non monotone dans le sens que la loi de monotonicité, i.e. la croissance linéaire des théorèmes à partir de l'ensemble des axiomes, ne tient plus puisque dans le premier cas le trop-plein de contenu informationnel déborde les capacités du moteur d'inférence, et dans le deuxième l'absence d'information stoppe ou déforme le mécanisme inférentiel dans le raisonnement par défaut.

La caractéristique principale d'une logique dynamique dialectique est la transitivité de la médiation < Vermittlung $>$, l'inférence propre à la syllogistique hégélienne. Je rappelle qu'une relation transitive $R$ signifie simplement en termes classiques

$$
x R y \text { et } y R z \rightarrow x R z
$$

C'est une relation d'équivalence si elle est en plus réflexive $(x R x)$ et symétrique $(y R x \rightarrow x R y$. Dans une logique monotone, l'inférence est bien entendu transitive, mais le caractère dynamique de la médiation au sens hégélien est perdu. La $<$ Vermittlung $>$ confère au moyen terme $<$ Mittel $>$ un rôle bien différent de celui qui lui est attribué dans le syllogisme aristotélicien "statique ", comme dans Barbara par exemple: 


\section{A est B \\ C est $A$ \\ C est $\mathrm{B}$}

où le moyen terme demeure un lien statique ou inerte dans les prémisses afin de réunir le petit terme et le grand terme dans la conclusion.

\section{Les syllogismes dynamiques}

Prenons trois exemples de syllogismes hégéliens tels qu'ils se trouvent à la fin de l'Encyclopédie ${ }^{6}$. Dans le premier syllogisme nous avons les trois termes, Logique <das Logische $>$, Nature et Esprit; le moyen terme est Nature.

$\begin{array}{ll}\text { Nature devient Esprit } & \mathrm{N} \rightarrow \mathrm{E} \\ \text { Logique devient Nature } & \mathrm{L} \rightarrow \mathrm{N} \\ \text { Logique devient Esprit } & \mathrm{L} \rightarrow \mathrm{E}\end{array}$

Il y a un passage ou une transition <ein Uebergehen $>$ de la Nature à l'Esprit, alors que dans le deuxième syllogisme, Esprit devient le moyen terme.
Esprit devient Logique
$\mathrm{E} \rightarrow \mathrm{L}$
Nature devient Esprit
$\mathrm{N} \rightarrow \mathrm{E}$
Nature devient Logique
$\mathrm{N} \rightarrow \mathrm{L}$

et finalement dans le troisième syllogisme, Logique ou Concept ou Idée < die Idee > est le moyen terme.

$\begin{array}{ll}\text { Logique devient Esprit } & \mathrm{L} \rightarrow \mathrm{E} \\ \text { Nature devient Logique } & \mathrm{N} \rightarrow \mathrm{L} \\ \text { Nature devient Esprit } & \mathrm{N} \rightarrow \mathrm{E}\end{array}$

Logique, Nature et Esprit s'échangent le rôle de moyen terme pour mettre en évidence le développement et l'évolution de l'activité du savoir $<$ die Tätigkeit des Erkennens>. Tout cela s'accorde avec la seconde thèse de la Dissertation de 1801: «Le syllogisme est le principe de l'idéalisme» et on pourrait avancer que la dialectique hégélienne est essentiellement une syllogistique dynamique. Il faut remarquer que dans les syllogismes que nous avons vus plus haut la transitivité n'est pas une relation d'équivalence, mais le procès du devenir ou un procès en devenir <ein Prozess im Werden>, comme le mathématicien intuitionniste L. E. J. Brouwer le disait à propos du continu mathématique. La nature dynamique de ce procès l'éloigne du caractère statique de la syllogistique aristotélicienne comme dans la règle d'inférence du Modus ponens:

$$
\frac{P \rightarrow \underset{P}{Q}}{Q}
$$


ou du Modus tollens:

$$
\begin{array}{r}
P \rightarrow Q \\
\neg Q \\
\hline \neg P
\end{array}
$$

Le départ de la conception traditionnelle est radical dans la pensée spéculative. Hegel écrit dans la première édition de la Wissenschaft der Logik :

Ist aber der Inhalt spekulativ, so ist auch das Nichtidentische des Subjects und des Prädikats wesentliches Moment, und der Uebergang oder das Verschwinden des ersten in das andere ihre Beziehung... Das wahre Resultat, das sich hier ergeben hat, ist das Werden, welches nicht bloss die einseitige oder abstracte Einheit des Seins und Nichts ist ${ }^{7}$ ( «mais si le contenu est spéculatif, l'est aussi le moment essentiel de la non-identité du sujet et du prédicat et le passage ou le disparaître du premier dans le second leur rapport même [...]. Le vrai résultat qui s'est produit ici est le devenir qui n'est pas seulement l'unité unilatérale et abstraite de l'être et du néant. »)

Le fait que le devenir n'est pas l'unité abstraite de l'être et du néant signifie qu'il y a ici un mouvement de sursomption de leur différence et en même temps de la relation d'équivalence qui constitue leur identité <identische Beziehung $>$. La copule qui relie le sujet et le prédicat dans la logique traditionnelle est cette relation d'équivalence qui ne va pas au-delà d'une identité sans vie. Mais comme Hegel le montre à la fin de la Wissenschaft der $\operatorname{Logik}^{8}$, le syllogisme de la méthode dialectique a une structure circulaire qui culmine dans le cercle des cercles <ein Kreis von Kreisen> décrivant le procès de la médiation dans la chaîne des déterminations du Concept lui-même. Ce qui fait défaut dans le syllogisme traditionnel, selon Hegel, c'est le procès dialectique de la négativité: la médiation médiatise l'immédiat dans un mouvement qui recrée le développement du Concept à travers la sursomption des étapes successives dans l'accession au savoir absolu. On peut résumer ce chapelet d'idées en disant que le vrai syllogisme de la dialectique hégélienne se révèle dans la transition dynamique de la sursomption d'une détermination à l'autre et non dans la relation statique de transitivité entre deux termes équivalents. La Phénoménologie de l'esprit exhibe déjà cette structure dynamique concrète.

\section{La Phénoménologie de l'esprit et la syllogistique dynamique}

La syllogistique dynamique est omniprésente dans la Phénoménologie de l'esprit, et je veux en marquer les points forts; je commence par la fin, avec le savoir absolu que j'ai quitté dans la Science de la logique:

7. Hegel, 6, p. 31.

8. Ibid., 7 . 
Er (der Gegenstand) ist, als Ganzes, der Schluss oder die Bewegung des Allgemeinen durch die Bestimmung zur Einzelheit, wie die umgekehrte, von der Einzelheit durch sie als aufgehobene oder die Bestimmung zum Allgemeinen. Nach diesen drei Bestimmungen also muss das Bewusstsein ihn als sich selbst wissen $^{9}$ ( «il (l'objet) est en tant que tout la conclusion ou le mouvement du général, ou la détermination vers la particularité, comme l'inverse, de la particularité en tant que sursumée ou la détermination vers le général. C’est selon ces trois déterminations que la conscience doit se connaître comme conscience de soi. »)

Ce passage résume toute la Phénoménologie dans sa structure logique. Le syllogisme est ici conçu comme le mouvement de l'universel vers le singulier à l'aide de la détermination et aussi bien comme le mouvement inverse du singulier à l'universel à l'aide de la sursomption ou de la médiation. Le facteur déterminant est le moyen terme en tant que médiateur tandis que l'universel et le singulier agissent comme les termes extrêmes, c'est-à-dire comme le petit terme et le grand terme. Hegel perçoit le grand terme et le petit terme comme les extrêmes entre lesquels se joue la contradiction, mais en réalité l'opposition ou la tension dialectique se situe entre termes contraires et sous-contraires, et non entre termes contradictoires d'après le carré d'Apulée :

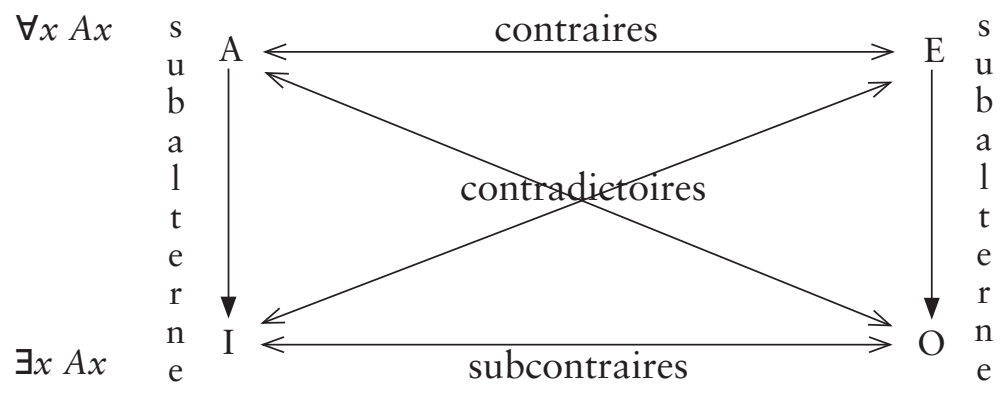

où nous avons :

$$
\begin{aligned}
& \forall x A x \equiv \neg \exists x \neg A x \\
& \forall x \neg A x \equiv \neg \exists x A x \\
& \exists x A x \equiv \neg \forall x \neg A x \\
& \exists x \neg A x \equiv \neg \forall x \quad A x
\end{aligned}
$$

Parcourant les figures (<Gestaltungen $>$ ) de la conscience, conscience immédiate, perception, entendement, conscience de soi, c'est le mouvement de la conscience elle-même qui accomplit la totalité de ses moments: Dies ist 
die Bewegung des Bewusstseins und dieses ist darin die Totalität seiner Momente $^{10}$. ( "C'est là le mouvement de la conscience, et elle est dans ce mouvement la totalité de ses moments.»)

Le mouvement de la conscience et le mouvement du Concept sont un seul et même mouvement, mais c'est seulement dans le savoir absolu que les deux mouvements convergent absolument, pour le dire en termes mathématiques. Le procès d'extériorisation <Entäusserung > rend possible l'automotion de la conscience et du Concept dans la triade immédiateté-médiationimmédiateté sursumée ou dans la triade en-soi-pour-soi-en-soi pour-soi $<$ Ansich--für sich-Ansich für sich>. Hegel a attribué la structure de la triplicité au syllogisme traditionnel d'Aristote à Kant, mais il a mis l'accent sur les limites de la structure formelle tout en insistant sur les propriétés spéculatives du syllogisme dialectique. Un exemple crucial est la genèse dialectique de la conscience de soi. Hegel écrit:

Die Mitte ist das Selbstbewusstsein welches sich in die Extreme zersetzt; und jedes Extreme ist diese Austauschung seiner Bestimmtheit, und absoluter Uebergang in das entgegengesetzte ${ }^{11}$. ( «Le milieu est la conscience de soi qui se divise en ses extrêmes; et chacun des extrêmes est cet échange de sa détermination et passage absolu dans l'opposé.»)

La réciprocité des deux opposés permet de concevoir chacun comme le moyen terme ou la médiation, et dans le procès de la médiation de se reconnaître l'un l'autre comme se reconnaissant mutuellement. Ce passage célèbre met en évidence la dialectique concrète du syllogisme dynamique et montre avec force comment la description phénoménologique épouse les règles de l'inférence dynamique en reflétant le mouvement ascendant de la conscience vers le savoir absolu, de la même manière que le développement formel de la logique (onto-logique) suit le mouvement du Concept dans son ascension vers l'Idée absolue.

\section{La logique dialectique}

J'ai tenté de montrer que la méthode dialectique de Hegel devait être interprétée comme une logique dynamique appliquée à la syllogistique traditionnelle et non en recourant aux approches formelles, algébriques ou non standard de la logique contemporaine; l'approche paraconsistante, par exemple, fait place à la contradiction dans un système formel et ne résout pas le problème de la transitivité. Je défends plutôt le programme consistant qui suppose que Hegel ne pensait pas en termes de contradictions formelles quand il écrivait dans la Dissertation de 1801: la contradiction est la règle pour le vrai <verum $>$ et la non-contradiction la règle pour le faux $<$ falsum $>$, mais qu'il pensait plutôt en termes de contraires «médiatisés" par un tiers 
inclus dans l'auto-développement du concept comme l'enseigne la Logique d'Iéna. Le concept <der Begriff $>$ est à la fois le milieu et le moyen terme <die Mitte $>$, le moyen même <das Mittel $>$ et la médiation <die Vermittlung $>$ du procès de la connaissance <das Erkennen $>$. La logique dialectique est la logique de l'action des concepts, de leur effectivité, pourrait-on dire < Tätigkeit und Wirkung des Begriffs>. Les contradictions sont des opposés, des extrêmes $<$ entgegengesetzte > ou des polarités contraires. Les contradictions formelles <in terminis > briseraient la chaîne des concepts de la même manière qu'elles coupent le lien inférentiel entre axiomes et théorèmes dans un système formel. En replaçant la logique dialectique dans le cadre syllogistique, on voit immédiatement que la logique du développement conceptuel n'obéit pas à la logique classique. Un exemple frappant là-dessus est le statut de la double négation <Negation der Negation> chez Hegel; ce n'est pas la négation booléenne qui est à l'œuvre ici, mais ce que j'appelle la négation locale. Dans la double négation hégélienne, la première joue le rôle d'une déterminité locale alors que la seconde la sursume: la double négation ne revient donc pas à l'assertion d'origine comme dans la double négation booléenne ou classique, mais constitue un nouvel énoncé engendré par le développement du concept. Le caractère processuel de cette double négation est manifeste dans le concept hégélien de la vraie infinité < die wahre Unendlichkeit>.

Il ne s'agit aucunement de présenter la logique hégélienne comme une logique non standard ou déviante susceptible de traitement formel. Comme chacun sait, la Science de la logique est une ontologie formelle plutôt qu'une logique formelle dans le sens moderne du terme. Néanmoins, le cadre d'une syllogistique dynamique suggère que Hegel a contribué de façon originale à l'histoire de la logique en construisant un nouveau régime pour la représentation philosophique du développement du savoir dans un moule traditionnel renouvelé.

\section{Conclusion}

\section{La syllogistique dynamique dans La science de la logique}

Dans le chapitre terminal de la Phénoménologie de l'esprit, Hegel résume le parcours de la conscience:

C'est là le mouvement de la conscience et celle-ci y est la totalité de ses moments ${ }^{12}$.

en parlant de l'esprit de la religion révélée qui n'a pas encore surmonté sa conscience comme telle - je suis ici la traduction de Jarczyk et Labarrière que je modifie à l'occasion. Hegel ajoute:

Ce n'est cependant pas le savoir comme acte pur de comprendre l'objet dont il est question, mais ce savoir doit seulement se trouver mis en évidence dans son devenir ou dans ses moments, selon l'aspect qui appartient à la conscience

12. Science de la logique, 10. p. 674. 
comme telle et les moments du concept proprement dit ou du pur savoir [mis en évidence] dans la forme de figurations de la conscience ${ }^{13}$.

Conscience et concept participent du même mouvement, et c'est dans la convergence de la série des moments du concept et des figures de l'esprit que s'accomplit le Savoir absolu où vérité et certitude correspondent parfaitement.

Les moments comme mouvements purs s'autopropulsant «indem diese [Momente] als die reine Bewegungen sich selbst weiter treiben ${ }^{14}$ " ne sont plus dans le Savoir absolu les figures déterminées de la conscience, mais plutôt les moments du mouvement du savoir "die Momente seiner Bewegung ", et ultimement ces moments s'identifient au moment même du concept pur qui ${ }^{15}$ prend la forme finale du cercle "der in sich zurückgehende Kreis"; l'Idée absolue, dans la conclusion de la Science de la logique, sera un cercle de cercles «Kreis von Kreisen» dans le mouvement circulaire de l'Esprit.

Faudra-t-il parler de moment angulaire et de commutations cycliques des moments de l'esprit dans ce contexte ? En tout cas, la syllogistique dynamique de Hegel suppose toujours ce mouvement de retour à soi :

[Le concept] se médiatise avec soi-même (mit sich selbst vermittelt) par sa négativité, du même coup est posé pour soi comme l'identité universelle de ses moments ${ }^{16}$.

La dialectique rotative des moments est le propre de la méthode spéculative de Hegel. Comme il le dit, la triplicité ou la quadruplicité n'est qu'un aspect superficiel ou abstrait de cette dialectique concrète, et s'il est vrai que «le syllogisme est le principe de l'idéalisme» comme il l'écrit dans la Dissertation de 1801, c'est que l'organisation du savoir en système repose sur la structure simple des trois termes (petit terme, grand terme et moyen terme) dynamisée par les moments du devenir ou l'auto-mouvement du concept.

Dès 1801, la réappropriation vitaliste de la mécanique newtonienne orientera toute l'entreprise hégélienne sous le paradigme du moment entendu comme moment cinétique ou moment angulaire. Que ce soit le concept physique d'une théorie mécanique sans vie - pour Hegel - qui soit à l'origine d'une métaphysique de la vie de l'esprit ne doit pas étonner le lecteur de Hegel. Le concept de vie est en effet le thème majeur des Écrits de jeunesse et la transition par le moment cinétique orbital, pourrait-on dire, de la dissertation De orbitis planetarum à la période de maturité ne pouvait s'opérer que par la sursomption du concept de vie dans la phénoménologie de l'esprit d'abord et dans la métaphysique ou l'onto-logique de l'idée absolue.

\section{7. Épilogue sur le vocabulaire hégélien}

Pour l'épistémologicien, le style et le vocabulaire de Hegel sont le propre de la prosopopée où le verbe pronominal accouplé au neutre du tiers (la troisième personne du singulier) constitue la trame du discours métaphysique qui enchaîne les noms inassignables (sans référence) dans une nomenclature ordonnée selon 
une sémantique spéculative. Mais la grammaire de ce discours est démontable (et non pas déconstructible, d'après une mauvaise traduction de l'allemand $<$ Abbau > que l'on trouve chez Heidegger dans son ouvrage Zur Seinsfrage [12] ). La consécution des moments (figures) de la conscience dans la Phénoménologie de l'esprit et la suite nécessaire des moments du concept dans La science de la logique ne constituent pas une syntaxe logique, mais plutôt la taxonomie des noms inassignables de l'histoire de la métaphysique. Le lien inférentiel est assez lâche qui va des Présocratiques à Hegel lequel reconstruit l'histoire conceptuelle de la métaphysique pour enfin clore le cercle de l'idée dans le circuit total des moments orbitaux, oserais-je dire. Lorsque Hegel met en jeu sa dialectique des extrêmes (Extreme), ce n'est rien d'autre qu'une syllogistique qui met en mouvement le terme majeur (le plus grand extrême) et le terme mineur (le plus petit extrême) par la médiation du moyen terme (Mitte) lequel devient précisément moyen (Mittel) de la médiation (Vermittlung) dans le milieu (Mitte) dynamique du devenir. La lexie ne doit pas nous tromper ici. Les commentateurs, comme B. Bourgeois, qui ne voient dans le vocabulaire de Hegel que «la langue la plus commune» ne pensent pas à lexicaliser le $<$ Moment $>$ hégélien (voir [1]). J'y vois plutôt l'irruption de la langue artificielle $<$ Kunstsprache $>$ qui vient corrompre subrepticement la langue commune, au point où cette immixtion peut passer inaperçue aux yeux d'un lecteur même averti. On ne saurait prétendre que les termes <Vermittlung $>,<$ Entäusserung $>$ ou $<$ Aufhebung $>$ tirent leur sème de la langue la plus commune. Traduire par exemple <Aufhebung > par "relève" à la suite de Derrida peut mener à des effets de style audacieux comme "le se relever" pour <das Sichaufheben $>$ de l'Esprit ou de la science à la fin de la Phénoménologie. L'amphibologie guette à tous les détours de la langue hégélienne. Un Joseph Gauvin, attentif au lexique de Hegel, à sa lettre et à son esprit, a introduit le terme extériorisation pour «Entäusserung» que d'aucuns rendaient par aliénation, confondant ainsi "Entfremdung» et "Entäusserung» (voir [5]). D'autres traducteurs bienveillants introduisent un vocabulaire étranger, p. ex. «l'être-là » heideggerien (Hyppolite) pour le $<$ Dasein $>$ hégélien qui veut simplement dire existence comment traduire <das Dasein Gottes $>$ sans tomber dans un absurde "être-là de Dieu ". D'autres encore (Jarczyk et Labarrière) s'adonnent à des excès de langage et des barbarismes comme «auto-conscience» (pour <Selbstsbewusstsein $>$ ) ou «autostance» (pour <Selbständigkeit $>$ ), alors que conscience de soi et autonomie font très bien l'affaire dans un paysage linguistique tout à fait familier pour Hegel. Quant au terme de sursomption que j'ai introduit, je ne chercherai pas à le défendre; d'autres l'ont fait (et mieux que moi), et s'ils ont réussi à imposer un lexème audacieux, ce n'est pas sans aller à une guerre ${ }^{17}$ où

13. Ibid., 10, p. 675.

14. Ibid., 7, p. 557.

15. Ibid., 7, p. 562.

16. Science de la logique, 11, p. 499. 
je n'ai pas été combattant, mais Jarczyk et Labarrière attribuent à qui de droit le terme de sursomption dans leur traduction de la Phänomenologie des Geistes $^{18}$.

La leçon ici n'est pas philosophique, puisqu'il n'est pas question de réduire un philosophème à un lexème, comme plus d'un serait tenté de le faire dans une sorte d'onomastique des noms inassignables de la métaphysique, "étymosophie ", science ou sagesse qui puise à l'origine des mots la richesse future de la pensée naissante. Ces jeux de langage ne sont pourtant pas étrangers à la pratique séculaire de la philosophie. D'autres savoirs, les mathématiques et la physique ou la biologie, même les sciences humaines et sociales, ont créé leur vocabulaire propre. Il revient au philosophe, qui partage ce privilège avec l'écrivain ou l'artiste, de créer sa langue propre au risque de ne plus y reconnaître sa propre langue"...

\section{Sources bibliographiques}

Bourgeois, B. Le vocabulaire de Hegel, Paris, Ellipses, 2000.

Gauthier, Y. La logique du contenu. Sur la logique interne. L'Harmattan, Paris, 2004.

—. "Logique hégélienne et formalisation», Dialogue, vol. VI, n 1967, p. 151165.

- - "Hegel's logic from a logical point of view", Hegel and the Sciences, Robert Cohen et Max W. Wartofsky, dir., Boston Studies in the Philosophy of Science, vol. 64, Reidel, Dordrecht, 1984, p. 303-310.

Gauvin, J. "Entäusserung et Entfremdung dans la Phénoménologie de l'esprit de Hegel ", Archives de philosophie, 1962, p. 555-571.

Hegel, G. W. F. Wissenschaft der Logik, Erster Band, Erstes Buch. Das Sein. Faksimiledruck nach der Erstausgabe von 1812, Vandenhock \& Ruprecht, Göttingen, 1966.

—. Wissenschaft der Logik, $2^{\mathrm{e}}$ Aufl. Erster Teil, Felix Meiner Verlag, Philosophische Bibliothek, Band 56, 1963.

-_. Jenenser Logik, Metaphysik und Naturphilosophie, Felix Meiner Verlag, Philosophische Bibliothek 58, Hamburg, 1957, p. 252 et ss.

—. Phänomenologie des Geistes, hrsg.v. J. Hoffmeister, Philosophische Bibliothek, vol. 114, Felix Meiner Verlag, Hamburg, 1952.

- - La phénoménologie de l'esprit, trad. G. Jarczyk et P.-J. Labarrière, Paris, Gallimard, 1993.

—. Enzyklopädie der philosophischen Wissenschaften im Grundrisse (1830), Felix Meiner Verlag, Hamburg, 1959.

Heidegger, M. Zur Seinsfrage, 1. Auflage, Frankfurt am Main, V. Klostermann, 1959.

Jarczyk, G. et P.-J Labarrière. Hegeliana, Paris, PUF, 1986.

Kant, I. Kritik der reinen Vernunft, Felix Meiner Verlag, Hamburg, 1956.

Newton, I. Mathematical papers, Éd. D.T. Whiteside, 8 volumes, Cambridge University Press, 1971.

17. Jarczyk et Labarrière, 13, p. 102-120.

18. Ibid., p. 60.

"Toutes les traductions sont de moi, à moins d'indications contraires. 